\title{
A Longitudinal Study of the Relations Between University Students' Subjective Social Status, Social Contact with University Friends, and Mental Health and Well-Being
}

\author{
Mark Rubin, Olivia Evans, and Ross B. Wilkinson \\ The University of Newcastle, Australia
}

This self-archived version is provided for non-commercial and scholarly purposes only.

The APA $\left(6^{\text {th }}\right.$ ed) style reference for this article is as follows:

Rubin, M., Evans, O., \& Wilkinson, R. B. (2016). A longitudinal study of the relations between university students' subjective social status, social contact with university friends, and mental health and well-being. Journal of Social and Clinical Psychology, 35, 722-737. doi: 10.1521/jscp.2016.35.9.722

We are grateful to Nicholas Harris and Gabriel Heaton for their assistance in recruiting research participants. This study was supported by a research grant from the Australian Government's Department of Education Higher Education Participation and Partnerships Programme National Priorities Pool. Correspondence should be addressed to Mark Rubin at the School of Psychology, The University of Newcastle, Callaghan, NSW 2308, Australia. Tel: +61 (0)2 4921 6706. Fax: +61 (0)2 4921 6980. Email: Mark.Rubin@ newcastle.edu.au 


\begin{abstract}
Prior research has found that the higher one's perceived status in society, the better one's mental health and well-being. The present research used a longitudinal design to investigate whether social contact with friends mediated this relation between subjective social status and mental health and well-being among first-year undergraduate students at an Australian university (Wave $1 N=749$, Wave $2 N=314$ ). Participants completed an online survey that included measures of subjective social status, social contact with university friends during the past week, and mental health and well-being during the past week. Multiple regression analyses found that subjective social status positively predicted amount of social contact with university friends, and that both of these variables positively predicted subsequent mental health and well-being. Furthermore, bootstrapped mediation tests found that social contact with university friends acted as a significant mediator of the relations between social status and mental health and well-being. These results are discussed in terms of their implications for the mental health and well-being of lower class students at university.
\end{abstract}

KEYWORDS: mental health, social class, social integration at university, socicoeconomic status, subjective social status. 
A Longitudinal Study of the Relations Between University Students' Subjective Social Status, Social Contact with University Friends, and Mental Health and Well-Being

Recent research has found that relatively large percentages of university students suffer from mental health problems. For example, in the USA, the 2015 National College Health Assessment II found that $18.6 \%$ of students reported having been diagnosed with depression (American College Health Association, 2015). This figure compares with only $6.7 \%$ in the USA general population (National Institute of Medical Health, 2015). Similarly, $19.2 \%$ of students at two large Australian universities had "very high" levels of mental distress (Stallman, 2010). This figure compares with only 3\% in the general population (Stallman, 2010). There is also longitudinal evidence that attendance at university worsens levels of depression and anxiety (Andrews \& Wilding, 2004). In the present research, we considered two variables that might predict better mental health and well-being among university students: subjective social status and social contact with university friends.

A person's subjective social status (SSS) reflects how they rank themselves relative to others in their community (Adler, Epel, Castellazzo \& Ickovics, 2000). SSS can be considered to be a psychological dimension of social class and socioeconomic status (e.g., Singh-Manoux, Marmot, \& Adler, 2005), and it has been shown to be positively related to mental health: The higher one's perceived social status, the better one's mental health and well-being (e.g., Cundiff, Smith, Uchino, \& Berg, 2013; Franzini \& Fernandez-Esquer, 2006; Goodman et al., 2001; Singh-Manoux, Adler, \& Marmot, 2003; Singh-Manoux et al., 2005). However, the process underlying this relation between SSS and mental health is unclear. In the present research, we considered social contact with friends as a potential explanatory (mediator) variable.

Social contact with friends has been found to be beneficial for mental health and wellbeing, most likely because it provides an important source of social support during stressful periods (for a review, see Hefner \& Eisenberg, 2009). Social contact with friends may mediate the relation between SSS and mental health at university because it is positively related to social class (Rubin, 2012; Rubin \& Kelly, 2015; Rubin \& Wright, 2015, in press). Consistent with this prediction, Rubin and Kelly (2015) recently found that general friendship and support and social integration at university mediated the relation between social class and mental health among a group of Australian undergraduate students. However, Rubin and Kelly's research suffers from three key limitations.

First, Rubin and Kelly (2015) assessed social class using measures of parental education, parental occupation, family income, self-reported social class identity, and house size. However, they did not make direct assessments of SSS. SSS is often a stronger predictor of mental health than other measures of social class and socioeconomic status (Adler et al., 2000; Singh-Manoux et al., 2005). Hence, the present research included a measure of SSS in order to determine whether it predicted mental health and well-being among university students.

Second, Rubin and Kelly (2015) assessed students' perceived friendship quality, social support, loneliness, sense of belonging, and community participation at university. Most of these measures represent psychological aspects of social integration, and they do not provide a direct assessment of social contact with other university students. It is important to investigate social contact because it represents a behavioural aspect of social integration that has tended to be omitted from investigations in this area. Hence, the present research also included a self-report measure of social contact with university friends.

Finally, as Rubin and Kelly (2015) conceded, a key limitation of their work is that it used a cross-sectional design. They recommended that future research use a longitudinal research design in order to reach firmer conclusions regarding the casual roles of the 
variables under consideration. The present research addressed this issue by using a crosslagged longitudinal research design.

\section{Participants}

\section{Method}

Participants were first-year domestic undergraduate students at a large publiclyfunded Australian university of around 40,000 students. Recruitment was restricted to firstyear undergraduate students because there is greater variability both between- and withinindividuals in the degree of social contact with friends at university during this commencement period. Students were recruited from across the university.

In Wave 1, data was collected from 1,211 students. Of these, 305 did not provide their informed consent and a further 157 were not first-year students, undergraduate students, domestic students, or non-psychology students. These 462 exclusions left a total of 749 participants. In Wave 2, data was collected from 500 students. Of these, 83 did not provide their informed consent and a further 103 did not meet the eligibility criteria for participation. These exclusions left a total of 314 students.

Rubin and Kelly's (2015) study of Australian undergraduate students found that the relation between social class and mental health had an effect size of $r=.15$. A power analysis showed that our Wave 2 sample of 314 participants had sufficient power (.76) to detect an effect of this size using a two-tailed correlation test with an alpha of level of .05.

We performed our analyses on data from the 314 students who completed both Waves 1 and 2 and who met the eligibility criteria. This sample included 202 women $(64.33 \%)$ and 112 men $(35.67 \%)$. Relative to the university population, the sample overrepresented women $(54.82 \%)$ and underrepresented men $(45.18 \%)$ by $9.51 \%$. To address this slight gender imbalance, gender was included as a covariate in all analyses. Participants ranged in age from 17 to 62 years with a mean age of $23.4(S D=7.86)$.

The majority of participants self-identified as Caucasian (89.5\%), with the remainder identifying as "other" (4.8\%), Aboriginal or Torres Strait Islander (3.5\%), Asian (1.6\%), or African (.6\%). Based on a measure of social class identity, $11.8 \%$ of participants described themselves as working class, $13.7 \%$ as lower middle class, $40.8 \%$ as middle class, $22.6 \%$ as upper middle class, $1.9 \%$ as upper class, and $2.88 \%$ selected "don't know."

Finally, $34.1 \%$ of our sample was enrolled in degrees in the Faculty of Education and Arts, $23.9 \%$ were from the Faculty of Health and Medicine, $15.0 \%$ were from the Faculty of Business and Law, 12.4\% were from the Faculty of Engineering and the Built Environment, $12.1 \%$ were from the Faculty of Science and Information Technology, and $2.5 \%$ did not identify a specific faculty.

\section{Procedure}

Wave 1 of data collection took place during Semester 1 (March - May 2015), and Wave 2 took place during Semester 2 (August - October 2015). The mean lag time between the two waves was 20.29 weeks, with the minimum and maximum lag times being 11.29 weeks and 28.71 weeks respectively.

Wave 1 was advertised via flyers and short presentations at lectures, and Wave 2 was advertised via email. Participation was voluntary and incentivized with entry into a prize draw for online gift certificates.

Participation was anonymous. The data from each wave of the survey were linked by means of a unique code that was generated by participants. The code consisted of their initials and their date of birth.

The research instrument consisted of a 15-minute online self-report survey titled "Your Relationships and Feelings during the Past Week." Participants completed the same 
survey during Waves 1 and 2. It included measures of subjective social status and social class, social contact and integration, mental health and well-being, university satisfaction, expected persistence at university, and alcohol and marijuana use. For the purposes of brevity and clarity, we focus on the measures of subjective social status, social contact, and mental health and well-being. A copy of the research survey, deidentified data set, and associated files are available at: https://bit.ly/2972paU

\section{Measures}

Subjective social status. Students' SSS was assessed using a modified version of the MacArthur Subjective Social Status scale (Adler et al., 2000). The scale is a single item that asks participants to rank themselves on an 11-point ladder diagram relative to other people in terms of their money, education, and occupation. In our modified version, we removed the ladder diagram and used a scale that was anchored bottom level and top level. Participants ranked themselves relative to other people in Australia. The SSS measure was placed near the end of the survey in order to mask its relevance to the measures of social contact and mental health and well-being (Langhout, Drake, \& Roselli, 2009).

Like Rubin and Kelly (2015), our survey also included measures of parental education, parental occupation, family income, and self-reported social class identity. These more objective measures of social class did not yield consistently reliable results in our analyses, and so we do not discuss them further.

Social contact with university friends. Social contact with university friends was measured using items that assessed the number of friends that students (a) currently had at university and (b) communicated with during the past week via (i) face-to-face meetings, (ii) email, (iii) social media, (iv) phone, and (v) text messages (Rubin \& Wright, in press). Responses were made on a scale ranging from 0 to more than 10. Communication via email was excluded from the final measure of social contact due to its detrimental impact on the internal reliability of the social contact measure. Participants also estimated the number of hours that they spent socializing with other students during the past week ( 0 to more than 20 ). The scores for these six social contact items were standardized in order to adjust for their different response scales and then averaged to form an overall index of social contact.

Like Rubin and Kelly (2015), our survey also included measures of friendship quality and quantity, loneliness, sense of belonging, and community participation. These more psychological (less behavioural) variables did not yield consistently reliable results in our analyses, and so we do not discuss them further.

Mental health and well-being. Following Rubin and Kelly (2015), mental health and well-being were measured using the short-form Depression Anxiety Stress Scale (DASS; Lovibond \& Lovibond, 2004) and the Satisfaction with Life Scale (SWLS; Diener, Emmons, Larsen, \& Griffin, 1985). The sort-form DASS is a 21-item measure of mental health over the past week. The scale is divided into three 7-item subscales that assess depression, anxiety, and stress. Example items are "I felt I wasn't worth much as a person" (depression), "I found it hard to wind down" (anxiety), and "I found it difficult to relax" (stress).

Participants responded using a 4-point scale ranging from never (0) to almost always (3).

The SWLS is a 5-item measure of well-being and life satisfaction. We modified the instructions to the scale and the items to refer to participants' life over the past week. Example items are "I was satisfied with my life during the past week," and "in most ways, my life during the past week was close to my ideal." Participants responded using a 7-point scale ranging from strongly disagree (1) to strongly agree (7). 


\section{Attrition Analyses}

\section{Results}

We conducted a series of independent-samples $t$ tests to compare the responses of participants who only completed Wave $1(N=435)$ with the responses of participants who completed both Waves 1 and $2(N=314)$. These tests were conducted on the key variables measured at Wave 1. There were no significant differences in SSS, DASS, depression, anxiety, stress, or SWLS. Additionally, there was no significant association between number of waves completed and either gender, faculty, or ethnicity. There was a marginally significant difference in age, $t(609)=-1.96, p=.050$. Participants who only completed Wave 1 were, on average, a year younger $(M=21.92, S D=6.63)$ than those who completed both Waves 1 and $2(M=22.98, S D=7.74)$. There was also a significant difference in social contact with university friends, $t(747)=1.99, p=.047$. Participants who only completed Wave 1 reported slightly more social contact $(M=0.05, S D=0.80)$ than those who completed both Waves 1 and $2(M=-0.07, \mathrm{SD}=0.76)$.

\section{Multiple Regression Analyses}

To assess the mediating effect of social contact with university friends in the relation between SSS and mental health and well-being, we tested the following four multiple regression models:

1. the effect of the Time 1 (T1) predictor variable (SSS) on Time 2 (T2) outcome variables (T2 DASS and T2 SWLS) controlling for T1 outcome variables (T1 DASS and T1 SWLS),

2. the effect of the T1 predictor variable (SSS) on the T2 mediator variable (T2 social contact with university friends) controlling for the $\mathrm{T} 1$ mediator variable (T1 social contact with university friends),

3. the effect of the T1 mediator variable (social contact with university friends) on T2 outcome variables (T2 DASS and T2 SWLS) controlling for T1 outcome variables (T1 DASS and T1 SWLS), and

4. the effect of the T1 predictor variable (SSS) on T2 outcome variables (T2 DASS and T2 SWLS) controlling for T1 outcome variables (T1 DASS and T1 SWLS) and the T1 mediator variable ( $\mathrm{T} 1$ social contact with university friends).

Tests 1,2, and 3 established the preconditions for a meaningful mediation effect: that SSS is a significant predictor of mental health and well-being (Test 1), and that the proposed mediator of social contact with university students is significantly related to both the predictor variable (Test 2) and the outcome variables (Test 3). Test 4 then established whether the size of the relations between SSS and mental health and well-being was reduced after controlling for the influence of social contact with university friends (the presumed mediator variable). Importantly, and unlike mediation tests in cross-sectional research designs, the current longitudinal approach measured prospective causal variables (SSS and social contact with university friends) at least 11 weeks earlier in time than outcome variables (mental health and well-being), thereby fulfilling a key criterion for establishing causation. In addition, our longitudinal tests of mediation controlled for the autoregressive effects of Time 1 instances of outcome/mediator variables, thereby reducing bias in the analyses due to the causal effects of prior levels of outcome variables (see Cole \& Maxwell, 2003, p. 562).

For all tests, multivariate outliers were excluded using a criteria of $p<005$. In addition, age, gender, ethnicity, and faculty were included as control variables. Given the very small numbers of participants in some ethnicity categories, ethnicity was coded as Caucasian (1) vs. other ethnicity (0). The inclusion or exclusion of (a) outliers and (b) control variables in our tests did not alter the pattern of significant results that are reported below. 
In Test 1, we regressed T2 mental health and well-being measures (DASS, depression, anxiety, stress, SWLS) onto T1 SSS controlling for corresponding T1 measures of mental health and well-being, age, gender, ethnicity, and faculty. T1 SSS was not a significant predictor of T2 DASS $(\beta=-.06, p=.163)$, T2 anxiety $(\beta=-.07, p=.110)$, or T2 stress $(\beta=-.03, p=.577)$. However, T1 SSS was a weak negative predictor of T2 depression $(\beta=-.10, p=.040)$ and a weak positive predictor of T2 SWLS $(\beta=.16, p=.002)$. Hence, SSS significantly predicted depression and satisfaction with life but not stress, anxiety, or overall DASS scores. Consistent with predictions, students who had lower SSS in Semester 1 had higher levels of depression and less satisfaction with life in Semester 2 even after controlling for Semester 1 depression and satisfaction.

In Test 2, we investigated whether T1 SSS predicted T2 social contact with university friends, controlling for T1 social contact, age, gender, ethnicity, and faculty. As expected, T1 SSS was a significant but weak predictor of T2 social contact $(\beta=.09, p=.024)$ : Students who had lower SSS in Semester 1 had less social contact with university friends in Semester 2 .

In Test 3, we investigated whether T1 social contact predicted the two measures of mental health and well-being that were predicted by T1 SSS, namely depression and SWLS. As before, we controlled for the relevant T1 mental health and well-being measures as well as $\mathrm{T} 1$ age, gender, ethnicity, and faculty. We found that T1 social contact was a weak negative predictor of T2 depression $(\beta=-.12, p=.014)$ and a weak positive predictor of T2 SWLS ( $\beta$ $=.13, p=.025)$. Hence, students who had relatively low levels of social contact with university friends in Semester 1 had higher levels of depression and less satisfaction with life in Semester 2 even after controlling for Semester 1 depression and satisfaction.

In Test 4, we investigated whether the effects of T1 SSS on T2 depression and SWLS became smaller after controlling for $\mathrm{T} 1$ social contact and the relevant $\mathrm{T} 1$ mental health and well-being measures, age, gender, ethnicity, and faculty. We found that the size of the effect of T1 SSS on T2 depression was smaller when controlling for T1 social contact $(\beta=-.08, p=$ $.081)$ than it was in Test 1 when the proposed mediator was uncontrolled $(\beta=-.10, p=.040)$. Similarly, the size of the effect of T1 SSS on T2 SWLS was smaller when controlling for T1 social contact $(\beta=.15, p=.006)$ than it was in Test 1 when the proposed mediator was uncontrolled $(\beta=.16, p=.002)$.

In summary, SSS in Semester 1 positively predicted social contact and satisfaction with life and negatively predicted depression in Semester 2. In addition, social contact with university friends in Semester 1 positively predicted satisfaction with life and negatively predicted depression in Semester 2. Finally, Test 4 showed that controlling for Semester 1's social contact with university friends reduced the size of the relation between Semester 1's SSS and Semester 2's depression and satisfaction with life. This pattern of results is consistent with the proposal that social contact with university friends mediates the relation between SSS and mental health (depression) and well-being.

\section{PROCESS Analyses}

Test 4 demonstrated that the total effects of T1 SSS on T2 depression and satisfaction with life were larger than the direct effects that controlled for T1 social contact. However, Test 4 did not test whether the size of these differences (i.e., the total effects minus the direct effects) were statistically significant. It is important to test the significance of these indirect effects in order to obtain more conclusive evidence of mediation. To this end, we employed Hayes' (2013) PROCESS macro. PROCESS uses a path analytical framework and bootstrapping to estimate direct and indirect effects in mediation models. We used PROCESS Model 4 to test mediation models in which T1 SSS was the predictor variable, T1 social contact was the mediator variable, and T2 depression and SWLS were the outcome 
variables. These models controlled for the relevant $\mathrm{T} 1$ outcome variable (i.e., $\mathrm{T} 1$ depression or T1 SWLS) as well as T1 age, gender, ethnicity, and faculty. In these models, we used 1,000 iterations to obtain the bias-corrected and accelerated bootstrap $95 \%$ confidence intervals.

When T2 depression was the outcome variable, the total effect of T1 SSS was significant, $B=-0.45, S E=0.22, p=.040,95 \% \mathrm{CI}(-0.873,-0.020)$, the indirect effect was significant, $B=-0.07, S E=0.03,95 \% \mathrm{CI}(-0.152,-0.013)$, and the direct effect was nonsignificant, $B=-0.38, S E=0.22, p=.081,95 \% \mathrm{CI}(-0.808,0.047)$. This pattern of results indicates that the relation between T1 SSS and T2 depression was fully mediated by T1 social contact with university friends.

When T2 SWLS was the outcome variable, the total effect of SSS was significant, $B=$ $0.13, S E=0.04, p=.002,95 \% \mathrm{CI}(0.048,0.214)$, the indirect effect was significant, $B=0.01$, $S E 0.006,95 \% \mathrm{CI}(0.001,0.025)$, and the direct effect was significant, $B=0.12, S E=0.04, p$ $=.004,95 \% \mathrm{CI}(0.039,0.205)$. This pattern of results indicates that the relation between SSS and satisfaction with life was partially mediated by social contact with university friends.

We also considered a reverse mediation model in which either depression or satisfaction with life mediated the relation between SSS and social contact with university friends. Contrary to these alternative models, neither T1 depression nor T1 SWLS were significant predictors of $\mathrm{T} 2$ social contact when controlling for T1 social contact, age, gender, ethnicity, and faculty (T1 depression: $B=-0.06, p=.122$; T1 SWLS: $B=-0.04, p=.366$ ). Hence, in the present study, social contact with university friends predicted mental health and well-being, but mental health and well-being did not predict social contact with university friends, making the reverse mediation model untenable.

\section{Discussion}

University students are at greater risk of mental health problems than the general population in both the USA and Australia (American College Health Association, 2015; Stallman, 2010). In the present research, we considered two variables that might predict better mental health and well-being among university students: subjective social status and social contact with university friends. In a sample of first-year Australian undergraduate students, we found that subjective perceptions of social status in society positively predicted amount of social contact with university friends, and that both of these variables negatively predicted depression and positively predicted well-being. Furthermore, social contact with university friends acted as a significant mediator of the relations between SSS and depression and satisfaction with life. These results are consistent with Rubin and Kelly's (2015) observation that social integration mediates the effects of social class on mental health and well-being. However, they build on this prior work by showing that Rubin and Kelly's results apply to (a) a measure of subjective social status and (b) social contact with university friends. In addition, the present research used a cross-lagged longitudinal research design rather than a cross-sectional design. Hence, we can be more confident than Rubin and Kelly that differences in SSS caused subsequent differences in (a) social contact with university friends and (b) mental health and well-being and that (c) differences in social contact caused subsequent differences in mental health and well-being. In particular, we found no evidence that differences in depression and satisfaction with life in Semester 1 predicted differences in social contact with university friends in Semester 2. Hence, it is more likely that social contact mediates the relations between SSS and mental health and well-being than it is that mental health and well-being mediate the relations between SSS and social contact.

Notably, the relations between SSS, social contact, and mental health were limited to depression and did not generalize to either anxiety or stress. The reasons for this divergence are unclear. However, it is possible that social contact is more reliably related to depression 
because depression is more of an interpersonal disorder than either anxiety or stress (e.g., Hammen, 1999). Consistent with this view, intervention evidence has found support for behavioral activation accounts of depression that emphasise the importance of social experiences as rewarding activities (Cuijpers, van Straten, \& Warmerdam, 2007).

\section{Limitations and Directions for Future Research}

Although the observed trends were in the predicted direction, our results did not generalize in a reliable manner to traditional proxies of social class and socioeconomic status (i.e., parental education, parental occupation, family income, and self-reported social class identity). This discrepancy reinforces the conclusions of previous research that subjective social status is a different and sometimes more powerful predictor of mental health than other measures of social class and socioeconomic status (Adler et al., 2000; Singh-Manoux et al., 2005).

The effects that we detected were not particularly large in size. The average size of the relation between T1 SSS and T1 and T2 measures of mental health and well-being was $r$ $=.18$, and the average size of the relation between $\mathrm{T} 1$ social contact and $\mathrm{T} 1$ and $\mathrm{T} 2$ measures of mental health and well-being was $r=.22$. Hence, these effects were small-to-medium in size. However, they are typical of the size of effects that have been found previously in this area (Rubin, 2012; Rubin \& Kelly, 2015; Rubin \& Wright, 2015, in press), and they are typical of the size of effects that tend to be found in psychology studies (Cafri, Kromrey, \& Brannick, 2010). Moreover, when extrapolated to the population level, these small-tomedium sized effects have the potential to affect thousands of students in higher education.

In general, participants who did not complete Wave 2 of our study were very similar on key variables to participants who did complete Wave 2. The exceptions were for age and social contact. Participants who did not complete Wave 2 were younger and had more social contact. This issue is unlikely to have had a substantial effect on our results and conclusions because we controlled for Wave 1 age and social contact in our analyses. Nonetheless, future research should carefully monitor and control for attrition effects and investigate the generalizability of our effects in populations that vary in age and degree of social contact.

A further limitation of our research is that our sample was restricted to first-year Australian undergraduate students from a single university. Future research in this area should attempt to replicate our findings among students at other stages of their university career, in other countries, and at other institutions. In addition, future research should explore the relations between subjective social status, social contact with friends, and mental health and well-being in non-educational contexts. For example, are similar relations observed in organisational contexts?

Finally, future research needs to probe the mediators and moderators of the relations between subjective social status, social contact with friends, and mental health and wellbeing. It is possible that social support may help to explain why social contact with friends is beneficial for mental health and well-being. In terms of moderator variables, it is possible that the type of friends with whom one has contact influences the degree to which contact improves mental health and well-being. In the middle-class environment of a university, contact with middle-class students may be more beneficial for mental health and well-being than contact with lower-class students. However, in more working-class environments, it is possible that the reverse is true. Future research should explore this context-based prediction.

\section{Implications}

The massification and diversification of higher education systems around the world has led to increases in the absolute numbers and relative proportions of students from lower class backgrounds at university. Our research findings suggest that these lower class students 
may be more depressed and less satisfied with their lives partly because they have less social contact with university friends. An obvious remedy to this problem is to increase amount of social contact that lower social class students have with other students at university. Rubin and Wright (2015, in press) recently discussed several intervention strategies for improving the social integration of working-class students at university, including subsidising the financial costs of (a) travel to university campuses, (b) on-campus and close-to-campus accommodation, (c) campus childcare, and (d) university-based social events. They also suggested online social networking as an effective form of social contact for time- and money-poor students. Based on the present research, all of these approaches are likely to increase social contact with university friends and, consequently, to improve the mental health and well-being of lower class students. A key challenge for future research in this area is to test these types of interventions and evaluate their effectiveness. 


\section{References}

Adler, N. E., Epel, E. S., Castellazzo, G., \& Ickovics, J. R. (2000). Relationship of subjective and objective social status with psychological and physiological functioning: Preliminary data in healthy, White women. Health Psychology, 19, 586-592. doi: 10.1037/0278-6133.19.6.586

American College Health Association. (2015). American College Health AssociationNational College Health Assessment II: Undergraduate students reference group data report Spring 2015. Hanover, MD: American College Health Association.

Andrews, B., \& Wilding, J. M. (2004). The relation of depression and anxiety to life-stress and achievement in students. British Journal of Psychology, 95, 509-521. doi: $10.1348 / 0007126042369802$

Cafri, G., Kromrey, J. D., \& Brannick, M. T. (2010). A meta-meta-analysis: Empirical review of statistical power, type I error rates, effect sizes, and model selection of metaanalyses published in psychology. Multivariate Behavioral Research, 45, 239-270. doi: 10.1080/00273171003680187

Cole, D. A., \& Maxwell, S. E. (2003). Testing mediational models with longitudinal data: Questions and tips in the use of structural equation modeling. Journal of Abnormal Psychology, 112, 558-577. doi: 10.1037/0021-843X.112.4.558

Cuijpers, P., van Straten, A., \& Warmerdam, L. (2007). Behavioral activation treatments of depression: A meta-analysis. Clinical Psychology Review, 27, 318-326. doi: 10.1016/j.cpr.2006.11.001

Cundiff, J. M., Smith, T. W., Uchino, B. N., \& Berg, C. A. (2013). Subjective social status: construct validity and associations with psychosocial vulnerability and self-rated health. International Journal of Behavioral Medicine, 20, 148-158. doi: 10.1007/s12529-011-9206-1

Diener, E. D., Emmons, R. A., Larsen, R. J., \& Griffin, S. (1985). The satisfaction with life scale. Journal of Personality Assessment, 49, 71-75. doi: 10.1207/s15327752jpa4901_13

Franzini, L., \& Fernandez-Esquer, M. E. (2006). The association of subjective social status and health in low-income Mexican-origin individuals in Texas. Social Science \& Medicine, 63, 788-804. doi: 10.1016/j.socscimed.2006.01.009

Goodman, E., Adler, N. E., Kawachi, I., Frazier, A. L., Huang, B., \& Colditz, G. A. (2001). Adolescents' perceptions of social status: Development and evaluation of a new indicator. Pediatrics, 108, e31-e31. doi: 10.1542/peds.108.2.e31

Hammen, C. (1999). The emergence of an interpersonal approach to depression. In T. Joiner \& J. C. Coyne (Eds.), The interactional nature of depression: Advances in interpersonal approaches (pp. 21-35). Washington, DC, US: American Psychological Association.

Hayes, A. F. (2013). An introduction to mediation, moderation, and conditional process analysis: A regression-based approach. New York: Guilford Press.

Hefner, J., \& Eisenberg, D. (2009). Social support and mental health among college students. American Journal of Orthopsychiatry, 79, 491- 499. doi: 10.1037/a0016918

Langhout, R. D., Drake, P., \& Rosselli, F. (2009). Classism in the university setting: Examining student antecedents and outcomes. Journal of Diversity in Higher Education, 2, 166-181. doi: 10.1037/a0016209

Lovibond, S. F., \& Lovibond, P. F. (2004). Manual for the Depression Anxiety Stress Scales. Sydney, NSW: The Psychology Foundation of Australia.

National Institute of Medical Health. (2015). Major depression among adults. Retrieved from http://1.usa.gov/1ElBJdO

Rubin, M. (2012). Social class differences in social integration among students in higher 
education: A meta-analysis and recommendations for future research. Journal of Diversity in Higher Education, 5, 22-38. doi: 10.1037/a0026162

Rubin, M., Denson, N., Kilpatrick, S., Matthews, K. E., Stehlik, T., \& Zyngier, D. (2014). "I am working-class:" Subjective self-definition as a missing measure of social class and socioeconomic status in higher education research. Educational Researcher, 43, 196200. doi: 10.3102/0013189X14528373

Rubin, M., \& Kelly, B. M. (2015). A cross-sectional investigation of parenting style and friendship as mediators of the relation between social class and mental health in a university community. International Journal for Equity in Health, 14(87), 1-11. doi: 10.1186/s12939-015-0227-2

Rubin, M., \& Wright, C. L. (2015). Age differences explain social class differences in students' friendship at university: Implications for transition and retention. Higher Education, 70, 427-439. doi: 10.1007/s10734-014-9844-8

Rubin, M., \& Wright, C. L. (in press). Time and money explain social class differences in students' social integration at university. Studies in Higher Education. doi: 10.1080/03075079.2015.1045481

Singh-Manoux, A., Adler, N. E., \& Marmot, M. G. (2003). Subjective social status: Its determinants and its association with measures of ill-health in the Whitehall II study. Social Science \& Medicine, 56, 1321-1333. doi: 10.1016/S0277-9536(02)00131-4

Singh-Manoux, A., Marmot, M. G., \& Adler, N. E. (2005). Does subjective social status predict health and change in health status better than objective status? Psychosomatic Medicine, 67, 855-861. doi: 10.1097/01.psy.0000188434.52941.a0

Stallman, H. M. (2010). Psychological distress in university students: A comparison with general population data. Australian Psychologist, 45, 249-257. doi: 10.1080/00050067.2010.482109 